\section{Ludger Schwienhorst-Schönberger}

Universität Wien

ludger.schwienhorst-schoenberger@univie.ac.at

DOI: http://dx.doi.org/10.12775/BPTh.2015.026

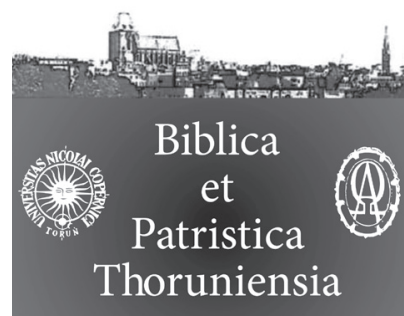

8 (2015) 4: 75-85

ISSN (print) 1689-5150

ISSN (online) 2450-7059

\title{
Josua, die Gewalt und die Bewohner des Landes
}

\section{Joshua, violence and the inhabitants of the land}

\author{
Jozue, przemoc i mieszkańcy ziemi
}

\begin{abstract}
The theological perspective of the events narrated in the book of Joshua is highlighted especially in their summary retrospect. It here becomes clear again that Israel's conquest of the land is no genocide motivated by religion. The decisive criterion is that of enmity towards Israel. "Enmity towards Israel", however, means "enmity towards an Israel which pays heed to God with all its heart and determines its conduct subject to the will of God." As soon as Israel resists the will of God, it prepares its own ruin. The decisive criterion is not ethnical, but ethical-theological in nature: it is a question of obedience or disobedience towards God. In Josh 21:44 we read: "Not one of their enemies had withstood them, for the LoRD had given all their enemies into their hands." Ultimately this means that resistance against God cannot be sustained indefinitely, be it displayed by Israel or by the peoples.

In its final composition, the book of Joshua assumes that Israel's enemies are an enduring reality. Israel nevertheless receives the assurance that it would be able to live in security (Josh 23:1-5; cf. Ps 23:5) even in the presence of these enemies. As such, the book sways within the field of tension between "already" and "not yet": YHWH had previously prevailed over Israel's enemies and had given the land to Israel. And yet the enemies remain a threat to Israel. Should Israel forget the covenant of its God, it would fall prey to the enemies (cf. Judg) and ultimately even be driven from the land (2 Ki 17; 25).

Thus the book of Joshua is not only, and not even primarily a book that looks back into the past, but one that looks towards the future, full of hope. To be exact: it is a book which develops criteria for a successful life in the present, consistent with godly standards, and which gains hope for a fulfilled future from this very life (Josh 23; 24).
\end{abstract}

Streszczenie. Teologiczna perspektywa wydarzeń opowiadanych w Księdze Jozuego jest uwypuklona zwłaszcza w ich podsumowaniach retrospektywnych. To tutaj jeszcze raz staje się jasne, że podbój ziemi przez Izrael nie jest ludobójstwem motywowanym religią. Decydującym kryterium jest wrogość wobec Izraela. „Wrogość wobec Izraela” oznacza jednak „wrogość wobec Izraela, który zwraca uwagę na Boga, z całego swego 
serca i określa swoje zachowanie zgodnie z wolą Boga”. Jeśli tylko Izrael odpiera wolę Boga, przygotowuje własną zgubę. Decydujące kryterium ma charakter nie etniczny, ale etyczno-teologiczny: jest to kwestia posłuszeństwa lub nieposłuszeństwa wobec Boga. W Josh 21:44 czytamy: „Żaden z ich wrogów nie mógł się wobec nich ostać, gdyż Pan wydał wszystkich ich wrogów w ich ręce." Ostatecznie oznacza to, że opór przeciwko Bogu, nie możne trwać w nieskończoność, jest on pokazany przez Izraela lub ludzi. W swojej ostatecznej kompozycji, Księga Jozuego zakłada, że wrogowie Izraela są trwałą rzeczywistością. Izrael jednak otrzymuje zapewnienie, że byłby w stanie żyć w bezpieczeństwie (Joz 23: 1-5; Ps 23: 5), nawet w obecności tych wrogów. Jako takie, księga znajduje się w polu napięć między „już i „jeszcze nie”: YHWH wcześniej pokonał wrogów Izraela i dał ziemię Izraelowi, jednak wrogowie pozostają zagrożeniem dla Izraela. Jeśli Izrael zapomni o przymierzu z Bogiem, to padnie ofiarą wrogów (por. Sdz) i ostatecznie może być wyprowadzony z ziemi (2 Krl 17; 25).

W ten sposób Księga Jozuego jest nie tylko, a nawet nie przede wszystkim, księgą, która patrzy w przeszłość, ale taką, który patrzy w przyszłość, jest pełna nadziei. Aby być dokładnym: jest to książka, która opracowuje kryteria udanego życia w teraźniejszości, zgodne z pobożnymi standardami, a które zyskuje nadzieję na spełnienie w przyszłości $\mathrm{z}$ tego prawdziwego życia (Joz 23; 24).

Keywords: Joshua (book of); Israel violence; settlement; Origen.

Słowa kluczowe: Jozue; Księga Jozuego; przemoc Izraela; porozumienie; Orygenes.

0 as Buch Josua gilt als ein schwieriges Buch. Der Historiker und Archäologe erhebt ihm gegenüber den Vorwurf, historisch unglaubwürdig zu berichten. Die Theologie beklagt die exzessive Gewalt, die darin im Namen Gottes gegenüber fremden Völkern ausgeübt wird. Ein Kritiker des Christentums wie Richard Dawkins sieht im Buch einen der vielen Belege für die Inhumanität des biblischen Gottes. Er schreibt: „Die ethnische Säuberung, die zu Zeiten Moses begann, findet ihren blutigen Höhepunkt im Buch Josua, einem Text, der durch die darin aufgezeichneten blutrünstigen Massaker ebenso auffällt wie durch seine genüsslich ausgebreitete Fremdenfeindlichkeit ... Und die biblische Geschichte über die Zerstörung Jerichos durch Josua sowie ganz allgemein über den Einzug ins Gelobte Land ist moralisch nicht von Hitlers Invasion in Polen oder Saddam Husseins Massakern an den Kurden und MarschArabern zu unterscheiden."1

In der Tat finden sich im Buch Josua Aussagen, die von der Eroberung des ganzen Landes und der Tötung all seiner Bewohner sprechen: „So schlug Josua das ganze Land - das Bergland und den Negeb, die Schefela und ihre Ausläu-

1 Richard Dawkins, Der Gotteswahn, s. 342, (s. 280). 
fer - mit allen seinen Königen. Niemand ließ er entkommen; alles, was lebte, weihte er dem Untergang, wie es der Herr, der Gott Israels, befohlen hatte" (Jos 10,40). Es finden sich aber auch gegenteilige Aussagen, die davon sprechen, dass Bewohner des Landes „bis auf den heutigen Tag“ mitten in Israel wohnen (Jos 6,25; 9). Eine diachron arbeitende Exegese versucht, diese einander widersprechenden Aussagen unterschiedlichen literarischen Schichten zuzuweisen. Ich halte diesen Ansatz prinzipiell für möglich und habe ihn bei der Analyse von Jos 6 angewandt. ${ }^{2}$ Auf der Ebene des Endtextes jedoch gilt, dass alle einander widersprechenden Aussagen „wahr sind“. Die Frage lautet: „Wahr“ - in welchem Sinne?

In einem längeren Aufsatz zum Thema „Josua 6 und die Gewalt“ habe ich die These vertreten, dass die Landnahmeerzählungen des Josuabuches letztlich in einem metaphorisch-paradigmatischen Sinn zu verstehen sind. ${ }^{3}$ Das metaphorisch-paradigmatische Verständnis ist in den Texten des Buches selbst angelegt. Es hat sich vor allem in der christlichen Auslegungsgeschichte weitgehend durchgesetzt. Das bekannteste Beispiel dürfte Origenes sein, der die Kämpfe, von denen das Josuabuch erzählt, als „innere, geistige Kämpfe“ versteht. Josua ist für ihn ein Typos für Jesus.

\section{Origenes: Der geistige Kampf}

Nach Origenes sind die Kriege, die Josua geführt hat, für Christen in einem geistigen Sinn zu verstehen. Der Kampfplatz ist im Inneren eines jeden Menschen zu suchen. Es handelt sich bei dieser Interpretation um ein hochgradig metaphorisches Verständnis der Texte, welches die in ihnen angelegte Tendenz der Entmilitarisierung bzw. Pazifizierung konsequent zu Ende denkt. Origenes schreibt: „Außer dir selbst sollst du außen kein Schlachtfeld suchen. In dir ist der Kampf, den du führen, im Inneren ist das morsche Gebäude, das du abreißen musst; dein Feind kommt aus deinem Herzen“ (Hom. 5,2). „In uns nämlich sind alle diese Völker von Lastern, welche die Seele dauernd und unablässig angreifen. In uns sind die Kanaanäer, in uns sind die Perisiter, hier sind die Jebusiter" (Hom. 1,7). Origenes begründet die Deutung mit Verweis auf den durch die christliche Rezeption erweiterten literarischen Kontext des Neuen Testaments. Hinzu kommt die Namensparallele „Josua - Jesus“; beide Namen sind im Griechischen identisch:'Inбoṽc. So kann Origenes weiterführend sa-

2 Vgl. Ludger Schwienhorst-Schönberger, Die Eroberung Jerichos.

3 Ludger Schwienhorst-Schönberger, Josua 6 und die Gewalt, in: Ed Noort (ed.), The Book of Joshua s. 433-471. 
gen: „Das ist nicht meine, sondern Christi Stimme; höre, wie er selbst sagt: ,Aus dem Herzen kommen die bösen Gedanken, Mord, Ehebruch, Unzucht, Diebstahl, falsches Zeugnis, Lästerungen' (Mt 15,19). Du siehst, welch großes Heer von Feinden in deinem Herzen gegen dich aufsteht. Ihnen müssen wir zuerst eine Niederlage beibringen, zuerst müssen wir sie niederwerfen. Wenn wir es geschafft haben, ihre Mauern zu Fall zu bringen und sie selbst dem Untergang zu weihen, so dass keiner von ihnen übrig bleibt (vgl. Jos 11,14), ... dann wird uns von Josua/Jesus die Ruhe geschenkt, so dass jeder unter seinem Weinstock und unter seinem Feigenbaum ausruhen kann, weil niemand mehr da ist, der die Kinder Israels aufschrecken könnte (vgl. Mi 4,4)“ (Hom. 5,2) ${ }^{4}$.

Bezüglich der Deutung des Josuabuches durch Origenes schreibt Thomas R. Elßner: „Letztlich besitzt es für Origenes keine wirkliche Relevanz mehr, ob die Erzählungen in Jos 6-8 geschichtliche Ereignisse wiedergeben. Deshalb verlässt er die Ebene des Literalsinns sogleich und wählt eine metaphorische Ebene mit geistlich-christlichem Gehalt. Letztere ist von bleibender Aussagekraft. Mehr noch: Der von Origenes entfaltete geistliche Gehalt ist von vornherein auch als primärer Schriftsinn intendiert. Nur missverständliches Lesen könne dann Texte wie Jos 6-8 allein als Wiedergabe exakter historischer Begebenheiten begreifen. Daher bewahrt der geistliche Sinn des Textes den sachkundigen Leser davor, dass ihn Texte wie Jos 6-8 zu höchst problematischen Handlungen provozierten. Jedoch hat Origenes auf seine Weise einerseits bereits erkannt, dass die biblischen Erzählungen des Josuabuches nicht mit historischen Berichten verwechselt werden dürfen. Andererseits betreibt er schließlich mit seinem hermeneutischen Vorverständnis eine Ethisierung der militärischen Kämpfe zur Einnahme des von Gott verheißenen Landes. Kampf ja, aber nur gegen die eigenen Sünden. Dem liegt eine Grundannahme zugrunde, nach der, wenn alle Christen gegen ihre eigenen Begierden und Laster vorgingen, die

4 Übersetzungen nach Thomas R. Elßner / Theresia Heither, Die Homilien des Origenes zum Buch Josua. In der lateinischen Übersetzung des Rufinus lautet das Zitat (hom. in Jesu Nave 5,1-2): „Praeter temet ipsum extrinsecus nihil requires. Intra te est proelium, quod gesturus es; intrinsecus est mala illa aedificatio, quae subruenda est; hostis tuus de corde tuo procedit. Non mea vox ista, sed Christi est; audi enim ipsum dicentem: De corde enim procedunt cogitations malae, homicidia, adulteria, fornications, furta, falsa testimonia, blasphemiae. Vides quantus et quails exercitus hostium tuorum adversum te de tuo corde procedit. Isti nobis prima strage fundendi sunt, isti prima acie prosternendi. Horum si subruere moenia ipsosque ad internitionem caedere potuerimus, ita ut non relinquamus ex eis ..., tunc nobis per Iesum dabitur illa requies, ut unusquisque requiescat sub vite sua et sub ficu sua, cum iam non sit, qui exterreat filios Israhel." 
christliche Gemeinde auch als ein Gegenentwurf gegenüber einer unbefriedeten Welt wahrgenommen und für diese attraktiv wird“ ${ }^{\text {" }}$.

\section{Israels Verhalten gegenüber den Bewohnern des Landes}

Im Folgenden möchte ich zeigen, dass ein metaphorisch-paradigmatisches Verständnis auch für das Verhältnis Israels zu den Bewohnern des Landes gilt. Die Bewohner des Landes repräsentieren unterschiedliche Verhaltensmodelle. Die Bewertung und die Folgen ihres Verhaltens messen sich vor allem an ihrem Verhalten zu Israel und vermittelt durch Israel an ihrem Verhalten zu Gott. Da die Erzählungen nicht in einem historischen Sinn zu verstehen sind, kommt ihnen eine metahistorische Bedeutung zu. Es geht also nicht um dieses oder jenes Volk, sondern um ein Verhalten, das von diesem oder jenem Volk repräsentiert wird. Damit lösen sich die Widersprüche auf, die sich bei einem historischen oder wörtlichen Verständnis ergeben. Zwar geht das Buch Josua davon aus, dass das hier erzählte Geschehen ein einmaliger Vorgang der Vergangenheit ist, doch diesem Geschehen kommt eine paradigmatische Bedeutung zu. Deshalb sind die Erzählungen im kulturellen Gedächtnis Israels aufbewahrt worden. Sie weisen eine Bedeutung auf, die „bis zum heutigen Tag“ (Jos 6,25) gilt. Beim Verhältnis Israels zu den Bewohnern des Landes kommen drei Modelle in den Blick: Ausrottung, Trennung, Integration.

Bevor die drei Modelle vorgestellt werden, sei daran erinnert, dass, genau genommen, das Land Israel gegeben wird. Die Aktivität Israels besteht darin, diese Gabe anzunehmen. So gesehen erzählt das Buch Josua nicht von der Landnahme Israels, sondern von der Langabe JHWHs und der Landannahme Israels. Diese Unterscheidung ist wichtig. Wo Israel versucht, eine Stadt eigenmächtig, auf eigene Initiative hin zu erobern, wie es bei der Stadt Ai der Fall ist, scheitert das Unternehmen (Jos 7). Die Einnahme des Landes durch Israel ist die der Gabe des Landes angemessene Form der Annahme. Sie ist nicht zu verwechseln mit einer eigenmächtigen Aneignung des Landes. Ich möchte im Folgenden zeigen, dass diese Struktur auch für das Verhältnis Israels zu den Bewohnern des Landes gilt. Die Stichworte Ausrottung, Trennung, Integration bezeichnen unterschiedliche Aspekte eines komplexen Vorgangs.

5 Thomas R. Elßner, Josua und seine Kriege, s. 253-254. 


\section{Ausrottung, Trennung und Integration bei der Eroberung Jerichos (Jos 6)}

Die Erzählung von der Eroberung Jerichos ist ein Beispiel für den passiven Widerstand, den eine Stadt gegenüber dem von JHWH angekündigten Geschehen leistet. Die Erzählung ist bekannt und oft ausgelegt und kommentiert worden. Ich selbst habe mich mit einer vor vielen Jahren erschienenen Studie daran beteiligt. ${ }^{6}$ Im Hinblick auf unsere Fragestellung ist der erste Satz der Erzählung von Bedeutung: „Jericho aber war verschlossen und wurde verschlossen. Niemand ging heraus, und niemand ging hinein" (Jos 6,1). Die Stadt leistet passiven Widerstand. Von sich aus ist Israel nicht in der Lage, diesen Widerstand zu brechen. In einem anschaulichen Bild wird erzählt, wie der Widerstand der Stadt durch ein von Gott gewirktes Wunder ,in sich zusammenfällt“: „Die Mauer fiel in sich zusammen, und das Volk ging hinauf in die Stadt, ein jeder an seiner Stelle, und sie nahmen die Stadt ein" (Jos 6,20). JHWH ist derjenige, der die Stadt und seinen König samt seinen Kriegern in die Hand Josuas gibt (Jos 2,2). Allerdings muss Israel diese Gabe auch annehmen. Und dies geschieht dadurch, dass es den Raum in Besitz nimmt.

Die Erzählung geht in Jos 6,1 davon aus, dass der Widerstand von der gesamten Bevölkerung der Stadt getragen wurde. Andernfalls wäre nicht zu verstehen, weshalb die gesamte Bevölkerung ausgerottet werden soll: „Mit scharfem Schwert weihten sie alles, was in der Stadt war, dem Untergang, Männer und Frauen, Kinder und Greise, Rinder, Schafe und Esel“" (Jos 6,21). Es gibt allerdings eine markante Ausnahme: die Hure Rahab und ihre Familie. Sie bleiben am Leben. Ich vermute, dass sich hier unterschiedliche Konzeptionen hinsichtlich der Bewohner des Landes niedergeschlagen haben. Auf die literarkritischen Probleme möchte ich hier nicht näher eingehen. Ich habe mich in anderen Zusammenhängen ausführlich dazu geäußert. Die Vernichtungsweihe geht wahrscheinlich auf eine ältere deuteronomistische Erweiterung zurück (DtrH und DtrP), während das Wohnenbleiben der Hure Rahab und ihrer Familie mitten in Israel auf eine spätere deuteronomistische Ergänzung (DtrN) zurückzuführen sein dürfte, die mit Texten wie Jos 23,5-13 und Ri 2,20-23; 3,4 übereinstimmt. Dort wird gesagt, dass ein Teil der Bevölkerung im Lande wohnen blieb, um Israels Gesetzesgehorsam zu erproben.

Hinsichtlich des Wohnenbleibens der Hure Rahab lassen sich zwei Modelle unterscheiden, die wahrscheinlich auf zwei unterschiedliche literarische Schichten zurückgehen. Die spät-deuteronomistische Erweiterung (DtrN)

6 Siehe Anmerkung 2. 
spricht einfach davon, dass Josua die Hure Rahab und ihre Familie am Leben ließ, so dass sie „mitten in Israel wohnt bis auf den heutigen Tag“" „Rahab aber, die Hure, und das Haus ihres Vaters und alle, die zu ihr gehörten, ließ Josua am Leben und so wohnt sie mitten in Israel bis auf den heutigen Tag, denn sie hat die Boten versteckt, die Josua geschickt hat, um Jericho auszukundschaften" (Jos 6,25).

Wahrscheinlich wird diese Sicht von einer priesterlichen Erweiterung differenziert. Sie fügt die Verse 22-23 hinzu: „Den zwei Männern aber, die das Land erkundet hatten, sagte Josua: ,Geht in das Haus der hurenden Frau und führt von dort die Frau heraus und alle, die zu ihr gehören, wie ihr es ihr geschworen habt.' Und die Diener, die erkundet hatten, gingen hinein und führten Rahab und ihren Vater und ihre Mutter und ihre Brüder und alle, die zu ihr gehörten, hinaus, (und all ihre Sippenverbände führten sie hinaus) und setzten sie außerhalb des Lagers Israel ab" (Jos 6,22f). Betont wird hier, dass die Hure Rahab „außerhalb des Lagers Israels abgesetzt wird“ - im Unterschied zu Vers 25, wo davon die Rede war, dass die Hure Rahab „mitten in Israel wohnt bis auf den heutigen Tag“. Hinter der priesterlichen Erweiterung, dass die Hure Rahab und alles, was zu ihr gehörte, „außerhalb des Lagers Israel abgesetzt wird", steht wahrscheinlich die theologische Konzeption von der Heiligkeit des Volkes Israel. Sie konkretisiert sich darin, dass sich das Volk von anderen Völkern abgrenzt. Diese Erweiterung dürfte in das 5. / 4. Jahrhundert zu datieren sein und die Diskussion um die Mischehen in den Büchern Esra und Nehemia widerspiegeln.

Die Endgestalt des vorliegenden Textes Jos 6 stellt uns drei unterschiedliche Modelle im Verhalten Israels zu den Bewohnern des Landes vor Augen: Ausrottung, Integration, Abgrenzung. Abhängig sind die unterschiedlichen Strategien vom Verhalten der Völker. Auf der einen Seite steht das Verhalten der Hure Rahab, auf der anderen Seite das Verhalten der übrigen Bewohner der Stadt Jericho. Die Botschaft lautet: Sich dem Handeln Gottes entgegenzustellen, hat auf Dauer keinen Bestand. Wer dagegen Gott und sein Handeln anerkennt, wie es die Hure Rahab tut, bleibt am Leben.

Wo genau die Hure Rahab am Leben bleibt, lässt der Text offen. Es lassen sich zwei Tendenzen erkennen: die eine geht in Richtung Integration („mitten in Israel“), die andere in Richtung Abgrenzung („außerhalb des Lagers Israels"). Diachron gesehen, aber auch vom Endtext her gelesen, hat das zweite Modell, die Abgrenzung, in diesem Fall die Oberhand gewonnen. Die Hure Rahab tritt nicht in das Gottesvolk ein, wie es bei der Moabiterin Rut der Fall ist. Und sie wird auch nicht „zwangsbekehrt“. Sie bekommt einen Platz „außerhalb des Lagers Israels“ zugewiesen (Jos 6,23). In diesem Sinne bleibt sie „mitten in Israel“" wohnen (Jos 6,25). 
Die Erzählung von der Eroberung Jerichos ist theologisch hoch aktuell. Der Glaube Israels führt nicht zu einer Ausrottung oder Zwangsbekehrung derjenigen, die nicht zum Gottesvolk gehören. Rahab darf als Nicht-Israelitin weiterhin mitten in Israel wohnen bleiben.

Dass die Vernichtung / Vernichtungsweihe (חרם) ganz auf Gott hin auszurichten ist, zeigt sich noch einmal daran, dass eine persönliche Bereicherung an dem, was Gott gehört, strengstens bestraft wird. ${ }^{7}$ In Jos 7,1 wird in nachholender Form erzählt, dass Achan, etwas von dem nahm, was dem Untergang geweiht war; er nahm sich etwas vom חרם. „Da entbrannte der Zorn des Herrn über Israel“, heißt es in Josua 7,1. Das macht noch einmal deutlich, dass die Erzählung in einem streng theologischen Sinn zu verstehen ist. Die dem Topos des Heiligen Krieges entlehnten Bilder wollen eine zutiefst theologische Wahrheit vermitteln: JHWH verschafft seinem Volk Lebensraum. Dieser Lebensraum geht aber nicht auf Kosten anderer. Wer dem Volk JHWHs diesen Lebensraum verweigert, geht zugrunde. In Analogie zum Verhalten der Rahab dürfen wir die Schlussfolgerung ziehen, dass die Bewohner der Stadt Jericho nicht der Vernichtungsweihe anheim gefallen wären, wenn die Stadt ihre Tore den heranrückenden Israeliten geöffnet hätte. Die Erzählung setzt implizit voraus, dass das Land genügend Platz für all seine Bewohner und für Israel bietet (vgl. Dtn 20,10-18).

\section{Die Eroberung der Stadt Ai (Jos 7-8)}

Die Erzählung von der Eroberung der Stadt Ai in Jos 7 ist eine Gegengeschichte zur Erzählung von der Eroberung Jerichos. Hier geht zunächst alles ganz anders aus. Josua handelt nicht auf Geheiß Gottes, sondern aus eigener Initiative: "Josua schickte von Jericho aus einige Männer nach $\mathrm{Ai}$... und sagte zu ihnen: Geht hinauf und erkundet das Land!“ (Jos 7,2). Sie handeln aufgrund eigenen Kalküls, wenn die Kundschafter sagen: „Es braucht nicht das ganze Volk hinaufzuziehen. Etwa zwei- oder dreitausend Mann sollen hinaufziehen; sie können Ai besiegen. Bemüh nicht das ganze Volk dorthin! Denn dort sind nur wenige Leute" (Jos 7,3). Was nach rein menschlichem Ermessen erfolgversprechend aussieht, endet in einem Desaster: Die zur Eroberung ausgesandten dreitausend Männer müssen die Flucht ergreifen. Das ganze Unternehmen der Landnahme droht zu scheitern. Die Botschaft lautet: Aus eigener Kraft kann Israel das Land nicht erobern.

7 Zum Thema „Bann“ vgl. Jannica A. de Prenter, The contrastive polysemous meaning, s. 473-488. 
Hinzu kommt ein Zweites: Wer sich etwas aneignet, was allein Gott zukommt, verunreinigt das Volk. Damit dringt eine Mentalität in das Volk ein, die den Sinn von Gott abwendet, die es dadurch schwächt und den Feinden ausliefert. Sie haben sich gegen den Bund vergangen (Jos 7,11). JHWH ist dann nicht mehr mit seinem Volk $(7,12)$. Das Volk selbst ist dann dem Untergang geweiht. Es bedarf der Reinigung (7,13: „Heiligung“). Das heißt, die Sünde / der Sünder muss aufgedeckt und vernichtet werden. Genau das geschieht in Jos 7,24-26.

In einem zweiten Anlauf gelingt die Eroberung Ais. Jetzt aber ist alles anders. JHWH ergreift die Initiative: „Und JHWH sprach zu Josua: Fürchte dich nicht und hab keine Angst! Nimm alle kriegstüchtigen Männer, brich auf und zieh nach Ai! Siehe, ich habe den König von Ai und sein Volk, seine Stadt und sein Land in deine Gewalt gegeben" $(8,1)$. Jetzt gelingt das Unternehmen. Die Stadt Ai wird erobert. Diesmal erteilt Gott die Erlaubnis, den Besitz und das Vieh der Stadt zu erbeuten $(8,2)$. Möglicherweise hängt das damit zusammen, dass die Männer von Ai die Israeliten aktiv bekämpft und beim ersten gescheiterten Versuch der Einnahme schweren Schaden zugefügt haben.

\section{Darbringung von Opfern und Verlesung des Gesetzes (Jos 8,30-35)}

Nach diesen beiden Modellen der Stadteroberung - genau genommen sind es drei Modelle (einmal Jericho, zweimal Ai) kommt es zu einem ersten Zwischenstopp: einer Opferliturgie in Verbindung mit der Verlesung des Gesetzes. Im Hinblick auf unsere Fragestellung ist die Beobachtung von Bedeutung, dass bei der Verlesung des Gesetzes „Fremde und Einheimische“ anwesend waren $(8,33)$. Zweimal wird gesagt, dass auch Fremde (גר), die mit ihnen zogen, dabei waren $(8,35)$.

\section{Integration auf der Grundlage eines Vertrages: die Gibeoniter (Jos 9)}

Im Folgenden wird das Thema: „Israel und die Bewohner des Landes“ um eine weitere Variante bereichert. Es kommt zu einem Vertrag zwischen Israel und einer Gruppe der Bewohner des Landes, nämlich den Gibeonitern. Israels $\mathrm{Zu}$ sammenleben mit den Gibeonitern wird vertraglich geregelt. Die Initiative geht dabei von den Gibeonitern aus. Sie verhalten sich ähnlich wie die Hure Rahab. Mit Hilfe einer List gelingt es ihnen, am Leben zu bleiben. Darin unterscheiden 
sie sich von den Königen der Hetiter, der Amoriter, der Kanaaniter, der Perisiter, der Hiwiter und der Jebusiter. Diese taten sich zusammen, um gemeinsam gegen Josua und Israel in den Kampf zu ziehen. Die weitere Erzählung wird zeigen, dass sie von Israel geschlagen werden (vgl. Jos 10ff).

Da die Gibeoniter sich aufgrund einer Täuschung einen Vertrag mit Israel erschlichen haben, wird ihnen eine untergeordnete Stellung im Land zugewiesen: Sie bleiben zwar am Leben, da der einmal unter Eid geschlossene Vertrag nicht gebrochen werden darf, aber sie werden zu „Holzfällern und Wasserträgern für die Gemeinde und für den Altar des Herrn gemacht“ $(9,26 f)$.

\section{Fazit}

Die theologische Perspektive des im Josuabuch erzählten Geschehens wird besonders in den summarischen Rückblicken hervorgehoben. Dabei wird noch einmal deutlich, dass es bei der Landnahme Israels nicht um einen religiös motivierten Genozid geht. Das entscheidende Kriterium lautet: Feindschaft gegenüber Israel. „Feindschaft gegenüber Israel“ heißt aber: „Feindschaft gegenüber einem Israel, das mit ganzem Herzen auf Gott hört und sein Verhalten vom Willen Gottes her bestimmt". Sobald sich Israel dem Willen Gottes gegenüber verweigert, bereitet es sich seinen eigenen Untergang. Das entscheidende Kriterium ist also kein ethnisches, sondern ein ethisch-theologisches. Es geht um Gehorsam und Ungehorsam gegenüber Gott. In Jos 21,44 heißt es: „Keiner von all ihren Feinden konnte ihnen Widerstand leisten. Alle ihre Feinde gab der Herr in ihre Gewalt." Letztlich bedeutet das: Widerstand gegenüber Gott hat auf Dauer keinen Bestand. Ob der Widerstand von Israel oder von den Völkern kommt, ist letztlich unerheblich.

In seiner Endkomposition geht das Buch Josua davon aus, dass die Feinde Israels eine bleibende Realität sind. Israel bekommt jedoch die Zusicherung, im Angesichte dieser Feinde sicher leben zu können (Jos 23,1-5; vgl. Ps 23,5). So gesehen bewegt sich das Buch in der Spannung von "Schon-und-Noch-nicht“: Gott hat die Feinde Israels einmal besiegt und Israel das Land gegeben. Dennoch bleiben die Feinde eine Gefahr für Israel. Wenn Israel den Bund seines Gottes vergisst, wird es den Feinden zum Opfer fallen (vgl. Ri) und letztlich sogar aus dem Land vertrieben werden (2 Kön 17; 25).

So gesehen ist das Buch Josua nicht nur, und nicht einmal in erster Linie, ein Buch, das in die Vergangenheit zurückschaut, sondern ein Buch, das voller Hoffnung in die Zukunft blickt. Genau genommen ist es ein Buch, das aus der Erinnerung an die Vergangenheit Kriterien für ein gelingendes, gottgemäßes 
Leben in der Gegenwart entwirft und daraus Hoffnung auf eine heilvolle $\mathrm{Zu}$ kunft schöpft (Jos 23; 24).

\section{Bibiography}

Ballhorn E., Israel am Jordan. Narrative Topographie im Buch Josua (BBB 162), Göttingen 2011.

Dawkins R., Der Gotteswahn, Berlin 2007 (The God Delusion, London: Transworld / Random House / Black Swan 2007).

Elßner Th. R., Josua und seine Kriege in jüdischer und christlicher Rezeptionsgeschichte (Theologie und Frieden 37), Stuttgart 2008, s. 253-254.

Elßner Th. R. - Heither T., Die Homilien des Origenes zum Buch Josua. Die Kriege Josuas als Heilswirken Jesu (Beiträge zur Friedensethik, 38), Stuttgart 2006.

Schwienhorst-Schönberger L., Die Eroberung Jerichos. Exegetische Untersuchung zu Josua 6 (SBS 122), Stuttgart 1986.

Schwienhorst-Schönberger L., Josua 6 und die Gewalt, in: Ed Noort (ed.), The Book of Joshua (BETL CCL), Leuven 2012, s. 433-471.

Schwienhorst-Schönberger L., Land, in: Michael Fieger / Jutta Krispenz / Jörg Lanckau (ed.), Wörterbuch alttestamentlicher Motive (WAM), Darmstadt 2013, s. 299-305.

de Prenter J. A., The contrastive polysemous meaning of חרם in the Book of Joshua, in: Ed Noort (ed.), The Book of Joshua (BETL CCL), Leuven 2012, s. 473-488. 